\title{
Redo surgery risk in patients with cardiac prosthetic valve dysfunction
}

Marek Maciejewski', Katarzyna Piestrzeniewicz ${ }^{1}$, Agata Bielecka-Dąbrowa², Monika Piechowiak', Ryszard Jaszewski

1Department of Cardiology, $1^{\text {st }}$ Chair of Cardiology and Cardiac Surgery, Medical University
of Lodz, Poland
2Department of Hypertension, Chair of Nephrology and Hypertension, Medical University
of Lodz, Poland
3Department of Cardiac Surgery, $1^{\text {st }}$ Chair of Cardiology and Cardiac Surgery, Medical
University of Lodz, Poland

Submitted: 8 June 2010

Accepted: 4 October 2010

Arch Med Sci 2011; 7, 2: 271-277

DOI: 10.5114 /aoms.2011.22078

Copyright @ 2011 Termedia \& Banach

\begin{abstract}
Introduction: The aim of the study was to analyse the risk factors of early and late mortality in patients undergoing the first reoperation for prosthetic valve dysfunction.

Material and methods: A retrospective observational study was performed in 194 consecutive patients ( $M=75, F=119$; mean age $53.2 \pm 11$ years) with a mechanical prosthetic valve ( $n=103$ cases; $53 \%)$ or bioprosthesis $(91 ; 47 \%)$. Univariate and multivariate Cox statistical analysis was performed to determine risk factors of early and late mortality.

Results: The overall early mortality was $18.6 \%$ : $31.4 \%$ in patients with symptoms of NYHA functional class III-IV and $3.4 \%$ in pts in NYHA class I-II. Multivariate analysis identified symptoms of NYHA class III-IV and endocarditis as independent predictors of early mortality. The overall late mortality ( $>30$ days) was $8.2 \%$ (0.62\% year/patient). Multivariate analysis identified age at the time of reoperation as a strong independent predictor of late mortality.

Conclusions: Reoperation in patients with prosthetic valves, performed urgently, especially in patients with symptoms of NYHA class III-IV or in the case of endocarditis, bears a high mortality rate. Risk of planned reoperation, mostly in patients with symptoms of NYHA class I-II, does not differ from the risk of the first operation.
\end{abstract}

Key words: prosthetic valve, reoperation, echocardiography.

\section{Introduction}

Artificial mechanical valves and bioprostheses which are implanted nowadays are highly durable and with little thrombogenicity [1-4]. However, redo surgeries in patients with implanted artificial valves are still a serious problem; this is so mainly because of the higher early mortality rate. In studies which have been published so far and which are based on analyses of a large amount of data [5-9], the authors usually presented the results of redo surgery performed during a period of about 30 years. A significantly higher early surgical mortality rate has already been reported before [7, $9,10]$. During the period included in the analysis, trans-oesophageal echocardiography was the basic method used in recognizing dysfunctions

\author{
Corresponding author: \\ Maciejewski Marek, MD, PhD \\ Department of Cardiology \\ $1^{\text {st }}$ Chair of Cardiology \\ and Cardiac Surgery \\ Medical University of Lodz \\ 1/3 Sterlinga \\ 91-425 Lodz, Poland \\ Phone/fax: +48 426364471 \\ E-mail: \\ marek56maciejewski@Gmail.com
}


of artificial valves and in qualifying for redo surgery treatments [11, 12].

Therefore the aim of our study was to analyse the risk factors of early and late mortality in patients undergoing a redo operation for prosthetic valve dysfunction.

\section{Material and methods}

\section{Patients' characteristics}

The material includes 194 successive patients with implanted artificial mechanical valves or with bioprostheses who were qualified to undergo a reoperation because of a valve prosthesis dysfunction between 1995 and 2006 in our department. Intraoperative diagnosis confirming the recognition was necessary for the patient to be included in the studied group. According to the proposition of Clark et al. [13], redo surgery was understood as reimplantation of a valve prosthesis which was implanted earlier.

The group of 194 patients contained 75 men and 119 women aged between 18 and 74 years (mean age $53.2 \pm 11$ years). The period between the implantation of an artificial valve and a redo procedure ranged from 6 days to 19 years. A hundred and twenty patients had an artificial mitral valve implanted (62 of them had a bioprosthesis) and 36 patients had an artificial aortic valve implanted (13 of them had a bioprosthesis). Thirty-five patients had both a mitral valve and an aortic valve implanted;
13 of them had two bioprostheses. Three patients had a biological valve and a mechanical valve implanted.

Twenty-two patients underwent redo surgery within 30 days from the implantation of an artificial valve. Forty-two redo procedures were performed as urgent indications. A hundred and five patients (54\%) were recognised as NYHA class III/IV. Atrial fibrillation was diagnosed in 145 patients. Structural dysfunction was the cause of redo procedure in 6 patients with a mechanical valve and in 76 patients with a bioprosthesis. Infectious endocarditis was recognised in 58 patients. In 30 patients with a mechanical valve a thrombus was the cause of blockage of the disc. Seventeen patients had a paravalvular shunt which was not connected with the ongoing endocarditis. Seven patients with mechanical valves underwent redo surgery because of other reasons (Table I). This time was the shortest for the infectious endocarditis. Thrombotic complications which occurred during the period between the implantation of an artificial valve and redo surgery were recognised in 17 patients.

\section{Protocol of the study}

When making a decision concerning redo surgery, the authors used the protocol adopted for recognising dysfunctions of artificial valves. If a dysfunction of an artificial valve was recognised on the basis of transthoracic examination, the decision concerning the resignation from transoesophageal examination was made after

Table I. Characteristics of the entire study group - 194 patients reoperated on for a prosthetic valve (PV) dysfunction

\begin{tabular}{|c|c|c|c|}
\hline \multicolumn{2}{|l|}{ Age [years] } & Mean (from-to) & $53.2 \pm 11(18-74)$ \\
\hline \multicolumn{2}{|l|}{ Sex } & Men (\%)/Women & $75(39) / 119$ \\
\hline \multicolumn{2}{|c|}{ Time from implantation of PV [months] } & Mean (from-to) & $83.4 \pm 65.6(1-230)$ \\
\hline \multirow[t]{3}{*}{ Reoperation: } & $\leq 30$ days & $n(\%)$ & $22(12.4)$ \\
\hline & $31-180$ days & $n(\%)$ & $38(19.6)$ \\
\hline & $>180$ days & $n(\%)$ & $132(68.0)$ \\
\hline \multicolumn{2}{|c|}{ Urgent reoperation } & $n(\%)$ & $42(21.7)$ \\
\hline \multicolumn{2}{|c|}{ Atrial fibrillation } & $n(\%)$ & $145(74.7)$ \\
\hline \multicolumn{2}{|c|}{ NYHA class III/IV } & $n(\%)$ & $105(54.1)$ \\
\hline \multicolumn{2}{|c|}{ PV mechanical/bioprosthesis } & $n(\%)$ & $103(53.1) / 91$ \\
\hline \multicolumn{2}{|c|}{ Embolic complications } & $n(\%)$ & $17(8.7)$ \\
\hline \multicolumn{4}{|c|}{ The reason for reoperation } \\
\hline \multicolumn{2}{|c|}{ Structural dysfunction of mechanical PV } & $n(\%)$ & $6(3.1)$ \\
\hline \multicolumn{2}{|c|}{ Structural dysfunction of bioprosthesis } & $n(\%)$ & $76(39.2)$ \\
\hline \multicolumn{2}{|c|}{ Infectious endocarditis } & $n(\%)$ & $58(29.9)$ \\
\hline \multicolumn{2}{|c|}{ Disc blockage by thrombus } & $n(\%)$ & $30(15.5)$ \\
\hline \multicolumn{2}{|c|}{ Paravalvular shunt } & $n(\%)$ & $17(8.8)$ \\
\hline \multicolumn{2}{|l|}{ Other* } & $n(\%)$ & $7(3.6)$ \\
\hline
\end{tabular}

*Temporal prosthetic valve dysfunction of unknown aetiology - 3 patients, pannus - 2 patients, "small" prosthetic valve - 2 patients, NYHA - New York Heart Association, PV - prosthetic valve 
consultation with a cardiac surgeon. In order to achieve the aims of this study, the authors analysed numerous preoperative clinical and echocardiographic parameters as well as surgical data.

\section{Cardiac surgical procedures}

In 152 patients the redo procedure involved only replacement of an artificial valve. In 42 patients aorto-coronary bridging or tricuspid annuloplasty was performed. Heart muscle protection was not significantly different in the patients undergoing redo surgery at the same time.

\section{Statistical analysis}

Continuous variables were expressed as the mean value $\pm S D$; Student's $t$-test was used to perform a comparative analysis [14, 15]. Categorical variables were expressed as percentages and the $\chi^{2}$ test or Fisher test was used for comparison [14, 15]. Results were recognised as statistically significant when $p<0.05$. A multifactorial analysis, the Cox proportional hazard models [16], was performed using the step method, separately for early mortality rate and late mortality rate. The Statistica software package was used for statistical analysis (StatSoft Poland).

\section{Results}

Early mortality ( $\leq 30$ days) in the patients who underwent redo surgery because of the dysfunction of an artificial valve (Table II) was $18.6 \%$ : $31.4 \%$ in patients with symptoms of NYHA functional class III-IV and 3.4\% in pts in NYHA class I-II. It was found that the following parameters have a significant influence on the result of a redo procedure: urgent indications ( $p<0.01)$, NYHA class III/IV $(p<0.001)$, infectious endocarditis (IE) $(p<0.001)$, redo surgery of an artificial mechanical valve $(p<0.005)$, kidney insufficiency ( $p<0.05)$, atrial fibrillation $(p<0.02)$, liquid in the pericardium $(p<0.05)$ and ejection fraction $(\mathrm{EF})<55 \%(p<0.01)$. The multifactorial analysis showed that the following were independent and significant negative prognostic factors: NYHA III-IV (odds ratio [OR] =3.89, $p=0.01$ ) and infectious endocarditis $(\mathrm{OR}=3.99, p<0.01)$.

The analysis of factors deciding about the results of redo surgery performed because of infectious endocarditis revealed the significant influence of the following parameters on the result of redo surgery: urgent indications $(p<0.02)$, NYHA III/IV $(p<0.001)$, time from the implantation of an artificial valve $(p<0.02)$, kidney insufficiency $(p<0.01)$, liquid in the pericardium $(p<0.05)$ and $\mathrm{EF}<55 \%(p<0.01)$. The multifactorial analysis showed that NYHA III-IV (OR = 4.02, $p=0.01)$ and ejection fraction below $55 \%(\mathrm{OR}=3.1, p<0.01)$ are significant independent negative prognostic factors.

In the group of 136 patients who underwent a redo procedure because of an artificial valve dysfunction caused by reasons other than infectious endocarditis (Table III) the following four significant

Table II. Clinical and echocardiographic predictors of death in early postoperative period in 194 patients reoperated on for prosthetic valve dysfunction

\begin{tabular}{|c|c|c|c|c|c|}
\hline \multicolumn{4}{|c|}{$\begin{array}{ll} & \text { Death }(n=36) \\
\end{array}$} & Survival $(n=158)$ & Value of $p$ \\
\hline Age [years] & \multicolumn{2}{|l|}{ Mean (from-to) } & $54.2 \pm 10.3,21-71$ & $53.0 \pm 11.3,18-74$ & NS \\
\hline Sex & \multicolumn{2}{|l|}{ Men/Women } & $12 / 24$ & $65 / 93$ & NS \\
\hline \multicolumn{3}{|c|}{ Urgent indications } & 14 & 28 & $<0.01$ \\
\hline \multicolumn{3}{|c|}{ NYHA class III-IV } & 33 & 72 & $<0.001$ \\
\hline \multicolumn{3}{|c|}{ Time from PVI [months] mean, med., from-to } & $45.9 \pm 63,8,1-224$ & $92.1 \pm 62,96,1-230$ & $<0.001$ \\
\hline & & $\leq 30$ days, $n$ & 11 & 14 & \\
\hline & & 31-180 days, $n$ & 20 & 17 & \\
\hline & & $>180$ days, $n$ & 5 & 127 & \\
\hline One valve & & $n$ & 30 & 129 & NS \\
\hline Two valves & & $n$ & 6 & 29 & NS \\
\hline PV mechanic & prosthesis & $n$ & $27 / 9$ & $76 / 82$ & $<0.005$ \\
\hline Additional $p$ & & $n$ & 9 & 33 & NS \\
\hline Infectious en & & $n$ & 14 & 44 & $<0.001$ \\
\hline Arial fibrillat & & $n$ & 33 & 112 & $<0.02$ \\
\hline Kidney insuf & & $n$ & 3 & 2 & $<0.05$ \\
\hline Liquid in the & rdium & $n$ & 7 & 6 & $<0.05$ \\
\hline Ejection frac & $55 \%$ & $n$ & 22 & 54 & $<0.01$ \\
\hline
\end{tabular}

med. - median, PVI - prosthetic valve implantation 
Table III. Clinical and echocardiographic predictors of perioperative death in patients referred for reoperation due to causes of prosthetic valve dysfunction other than endocarditis

\begin{tabular}{|c|c|c|c|c|c|}
\hline & & & Death $(n=22)$ & Survival $(n=114)$ & Value of $p$ \\
\hline Age [years] & \multicolumn{2}{|l|}{ Mean (from-to) } & $54.57 \pm 8.7(38-69)$ & $53.91 \pm 10.7(18-73)$ & NS \\
\hline Sex & \multicolumn{2}{|l|}{ Men/Women } & $4 / 18$ & $40 / 74$ & NS \\
\hline \multicolumn{3}{|c|}{ Urgent indications } & 5 & 20 & NS \\
\hline \multicolumn{3}{|c|}{ NYHA class III-IV } & 20 & 48 & $<0.001$ \\
\hline \multicolumn{4}{|c|}{ Time from PVR [months] mean, med., from-to } & $105.9 \pm 60,114,0-230$ & $<0.02$ \\
\hline & & $\leq 30$ days; $n$ & 7 & 8 & \\
\hline & & 31-180 days; $n$ & 12 & 7 & \\
\hline & & $>180$ days; $n$ & 3 & 99 & \\
\hline One valve & & $n$ & 20 & 93 & NS \\
\hline Two valves & & $n$ & 2 & 21 & NS \\
\hline PV mechani & prosthesis & $n$ & $15 / 7$ & $45 / 69$ & $<0.02$ \\
\hline Additional $p$ & ures & $n$ & 6 & 23 & NS \\
\hline Prosthetic v & ysfunction* & $n$ & 15 & 45 & - \\
\hline Disc blockag & & $n$ & 7 & 23 & NS \\
\hline Paravalvular & & $n$ & 5 & 12 & NS \\
\hline Structural d & ration & $n$ & 2 & 4 & NS \\
\hline Other & & $n$ & 1 & 6 & NS \\
\hline Atrial fibrilla & & $n$ & 21 & 84 & $<0.01$ \\
\hline Ejection frac & $55 \%$ & $n$ & 11 & 40 & NS \\
\hline
\end{tabular}

PVI - prosthetic valve implantation, *refers to patients operated on for mechanical prosthetic valve dysfunction

negative prognostic factors were distinguished: NYHA III-IV ( $p<0.001)$, time from the implantation of an artificial valve $(p<0.02)$, redo surgery of a mechanical valve $(p<0.02)$ and atrial fibrillation $(p<0.01)$. The multifactorial analysis showed that NYHA III-IV (OR $=1.58, p<0.05)$ is the only significant independent negative prognostic factor.

The comparative analysis of the patients who underwent redo surgery because of infectious endocarditis and because of other reasons is presented in Table IV. It revealed that men significantly outnumbered women in the IE group $(p<0.01)$ and that there is no significant difference between the ages of the patients undergoing redo surgery. Redo procedures due to reasons other than IE were performed significantly later (median 109 months, for IE 15 months, $p<0.001$ ). The analysis of the direct cause of redo surgery in patients with an implanted mechanical valve showed that valvular shunt occurred significantly more often

Table IV. Characteristics of patients reoperated on for prosthetic valve (PV) dysfunction due to infective endocarditis $(\mathrm{IE}+)$ and other reasons (IE -)

\begin{tabular}{|c|c|c|c|c|}
\hline & & $\mathrm{IE}+(n=58)$ & $\mathrm{IE}-(n=136)$ & Value of $p$ \\
\hline Age [years] & Mean (from-to) & $51.43 \pm 12.49(21-74)$ & $54.0 \pm 10.4(18-73)$ & NS \\
\hline Sex & Men/Women & $31 / 27$ & $44 / 92$ & $<0.01$ \\
\hline \multicolumn{2}{|c|}{ Time from PVI [months] mean, med., from-to } & $49.3 \pm 56,15,1-190$ & $98.9 \pm 64,109,1-230$ & $<0.001$ \\
\hline Bioprostheses & $n(\%)$ & $13(22.4)$ & $76(55.9)$ & $<0.001$ \\
\hline \multicolumn{5}{|c|}{ Direct cause of mechanical PV reoperation } \\
\hline Disc blockage & $n(\%)$ & $16(35.6)$ & $30(50.0)$ & NS \\
\hline Paravalvular leak & $n(\%)$ & $24(53.3)$ & $17(28.3)$ & $<0.02$ \\
\hline $\mathrm{DB}+\mathrm{PL}$ & $n(\%)$ & $5(11.1)$ & - & - \\
\hline Other & $n(\%)$ & - & $13(21.7)$ & - \\
\hline
\end{tabular}

med. - median, IE - endocarditis, PVI- prosthetic valve implantation 
in the patients with IE. Co-occurrence of valvular shunt and blockage of a heart valve disc was observed only in patients with IE. The patients with an implanted bioprosthesis constituted a significantly smaller subgroup among the patients who underwent redo surgery because of IE $(p<0.001)$.

Late results ( $>30$ days) in the patients who underwent redo surgery because of the dysfunction of an artificial valve amounted to $8.2 \%$. The observation time ranged from 4 to 132 months, 63 months on average; the total observation time amounted to 749.5 years. Late mortality rate was $0.62 \%$ per patient-year. It was revealed that the following parameters had a significant influence on the late result of redo surgery: a patient's age at the moment of redo surgery $(p<0.01)$, male sex $(p<0.01)$, redo procedure of a mechanical valve $(p<0.05)$ and atrial fibrillation $(p<0.05)$. The multifactorial analysis showed that the age of a patient at the moment of redo surgery was an independent and significant negative prognostic parameter $(\mathrm{OR}=2.29, p<0.05)$. Nineteen patients (12\%) underwent another redo procedure, including 5 patients $(26.3 \%)$ who died during the perisurgical period.

\section{Discussion}

Structural dysfunction of a mechanical valve was a cause of redo surgery in 3\% of the patients. A similar percentage of structural dysfunctions was observed by Bortolotti et al. in a group of 549 patients who underwent a redo procedure within a period of 26 years [11]. In the present study, the most frequent cause of a redo procedure was structural dysfunction of a bioprosthesis (76 patients - 39.2\%). Other authors indicate degeneration of a bioprosthesis as the most frequent cause of redo surgery as well [7, 17]. Each patient whose survival time is long enough needs redo surgery and this is connected with the limited durability of a bioprosthesis [18]. Taking that into account, it is proposed that implantation of bioprostheses be limited to patients over the age of 65. Summing up the results of a 15-year randomized study which compared bioprostheses and mechanical valves, Hammermeister et al. [19] stated that redo procedures occurred significantly more frequently only in a group of patients below the age of 65 . In the presented material, a direct indication for redo surgery in a majority of the patients was valve incompetence $(77.6 \%)$ and only in 17 cases was it the stenosis of a bioprosthesis. Only 5 patients underwent redo surgery urgently; the other ones underwent a redo procedure according to the schedule. These patients were re-examined every 3-6 months within the last year before the redo surgery. The diagnostic procedure which was used and the percentage of patients who underwent a redo procedure according to the schedule did not differ from other authors' reports [17].

In the present study, the early mortality rate was $18.6 \%$. Other authors reported mortality rates from $6 \%$ to $41 \%[7,9,10,20-22]$ depending on many factors connected with the clinical condition of a patient, advancement of valve pathology and the surgical procedure used.

This research confirmed the earlier observations $[5,14,22,23]$ concerning the negative prognostic influence of NYHA class III-IV and urgent redo surgery performance on the perisurgical mortality rate. In the present study, the mortality of patients who underwent redo surgery in NYHA class III/IV was $31.4 \%$ compared to $3.4 \%$ in NYHA class I/II. Similarly, for redo procedures performed urgently it was 33\% and for scheduled treatments $14.5 \%$. Urgent recommendations were not a negative factor in patients undergoing redo procedures because of valvular dysfunction resulting from reasons other than infectious endocarditis (Table III). However, redo surgery cannot be treated as an independent negative prognostic factor because a majority of patients treated urgently were in NYHA class IV. In the group presented by Husebye et al. [15] only one of 223 patients undergoing scheduled redo surgery and $64 \%$ of patients undergoing a redo procedure urgently were in NYHA class IV. These authors assessed the risk of redo surgery performed according to schedule as $1.3 \%$. In the present study, 33\% of patients undergoing scheduled redo surgery were in NYHA class III/IV. These differences might have resulted in a higher mortality rate in the perisurgical period. Like the redo procedure, the time which elapsed from the first surgery is related to the functional condition. Patients who underwent redo surgery up to 180 days after the implantation of a valve were usually in NYHA class III/IV and in those cases the results of redo surgery were worse (Tables II-IV).

Structural dysfunction of a bioprosthesis does not increase the perisurgical risk if the surgery is performed according to schedule [24]. In the present study, the mortality rate was lower by $10 \%$ (71 out of 76 patients underwent scheduled redo procedures). The opinions of other authors are similar [7, 25, 26].

Infectious endocarditis usually [27] significantly increases the risk of perisurgical death (from $24 \%$ to $62.5 \%)$. In the present study, the mortality rate was only $24 \%$, although all patients were in NYHA class III/IV and 9 out of 14 treated patients underwent redo surgery urgently. The multifactorial analysis revealed that infectious endocarditis is an independent factor which is prognostically negative.

Additional redo procedures are burdened with an increasing perisurgical mortality rate. The risk of the second redo surgery is only slightly higher 
[22] but the risk of the third redo surgery and of subsequent ones is significantly higher at $45 \%$ [27]. Similarly, in the present study, the mortality rate because of further redo surgery was only slightly higher at $26.3 \%$.

In the present study, ejection fraction lower than $55 \%$ was a negative prognostic factor in perisurgical mortality only in the group of patients with infectious endocarditis (Tables II, IV). It seems that it is mainly connected with quickly increasing valvular shunt, which haemodynamically corresponds to acute mitral or aortic incompetence. Turina et al. [28] did not find that a decrease in ejection fraction $<50 \%$ had an influence on perisurgical mortality in patients with an artificial valve dysfunction which led to valvular stenosis. However, they observed a tendency (not statistically significant) towards worsening of redo surgery results in patients with a dysfunction which led to mitral or aortal incompetence. Other analysed echocardiographic factors were not prognostically influential or were closely connected with functional condition (atrial fibrillation, kidney insufficiency or liquid in the pericardium).

Late mortality occurred in $0.62 \%$ of patients per year. The only independent factor which was prognostically negative was the age of the patients at the moment of redo surgery. Other authors have pointed to the importance of population factors [21, 27] and the functional condition (NYHA class IV) [7, 8]. In the opinion of Edwards et al. [29], the factors which negatively influence the late results of redo procedures performed because of infections of an artificial valve were the age of the patient and the replacement of a biological valve with a mechanical one.

In conclusion, redo procedures of patients with implanted artificial valves performed urgently, particularly in patients in NYHA class III/IV, and caused by infectious endocarditis, are burdened with a very high risk. However, the risk of scheduled redo surgery, particularly in patients in NYHA class I/II, does not differ from the risk of the first surgery which is implantation of a valve. Urgent redo surgery, burdened with a high risk, is necessary only in patients in NYHA class III/IV with valvular thrombosis, which results in the blockage of a valve, and with contraindications to thrombolytic treatment, as well as in patients with structural dysfunction of a mechanical valve or infectious endocarditis. Redo procedures in those patients should be performed as early as possible in order to avoid complications, particularly systemic embolism and cardiogenic shock. Other patients' condition usually improves after medical treatment which, most frequently, makes it possible to change the redo surgery qualification from urgent to scheduled. It is also important to distinguish patients with higher risk of redo surgery from patients with implanted artificial valves. This concerns particularly bioprostheses and some mechanical valves as well as patients with recognised "mild valvular shunt".

According to generally accepted standards [15, 30], there are no recommendations for routine echocardiographic examinations in patients with implanted artificial mechanical valves. However, it should be considered to provide patients with valves of "an old type", particularly bell and cage valves and some disc valves [20, 25, 28] as well as patients with high risk of thrombotic-embolic complications with such examinations. Patients with "mild valvular shunt" also need routine examinations because the degree of their illness may increase [26]. Unlike cases with mechanical valves, there is a common consensus of opinion [15, 30] that it is necessary to monitor patients with implanted bioprostheses using echocardiography, particularly if the first symptoms of valve degeneration appear.

The aim of echocardiographic examination in patients with valvular thrombosis which causes blockage of the valve is to single out those cases where the probability of medical treatment effectiveness is low and which require urgent reoperation. Echocardiographic examination is of particular significance in patients with dysfunction of an artificial valve caused by infectious endocarditis [31].

The present study has some limitations. The study group contained a small number of patients with old generation valves in which structural dysfunctions occurred more frequently. The majority of valves implanted nowadays are free from this complication. It is difficult to compare results presented by various authors because of differences concerning the analysed periods, material, methodology and lack of a common definition for redo procedure which would be accepted by all authors.

The research which was carried out and the analysis of the literature make it possible to propose an algorithm for treatment in patients qualified for reoperation because of valve dysfunction based mainly on echocardiographic assessment.

\footnotetext{
References

1. Aagaard J, Tingleff J, Hansen CN, et al. Twelve years' clinical experience with the CarboMedics prosthetic heart valve. J Heart Valve Dis 2001; 10: 177-84.

2. Jones JM, O'Kane H, Gladstone DJ, et al. Repeat heart valve surgery: Risk factors for operative mortality. J Thorac Cardiovasc Surg 2001; 122: 913-8.

3. Karalis DG, Chandrasekaran K, Ross JJ Jr, et al. Single-plane transesophageal echocardiography for assessing function of mechanical or bioprosthetic valves in the aortic valve position. Am J Cardiol 1992; 69: 1310-5.
} 
4. Pansini S, Ottino G, Forsennati PG, et al. Reoperations on heart valve prostheses: an analysis of operative risks and late results. Ann Thorac Surg 1990; 50: 590-6.

5. Antunes MJ, Magalhaes MP, Azevedo MG, et al. Reoperations of the mitral and aortic valves. Z Kardiol 1986; 75: 163-7.

6. Bortolotti U, Guerra F, Magni A, et al. Emergency reoperation for primary tissue failure of porcine bioprostheses. Am J Cardiol 1987; 60: 920-1.

7. Bortolotti U, Milano A, Mossuto E, et al. The risk of reoperation in patients with bioprosthetic valves. J Card Surg 1991; 6: 638-43.

8. Caus T, Albertini JN, Chi Y, et al. Multiple valve replacement increases the risk of reoperation for structural degeneration of bioprostheses. J Heart Valve Dis 1999; 8 : 376-83.

9. Chambers J. Echocardiographic assessment of artificial heart valves: British Society of Echocardiography position paper. Br Heart J 1994; 71: 6-14.

10. Kawachi Y, Matuzaki K, Tominaga R, et al. The risks of reoperation for prosthetic valve dysfunction. Surg Today 1994; 24: 415-9.

11. Kaymaz C, Ozkan M, Ozdemir N, et al. Spontaneous echocardiographic microbubbles associated with prosthetic mitralvalves: mechanistic insights from thrombolytic treatment results. J Am Soc Echocardiogr 2002; 15: 323-7.

12. Nitter-Hauge S, Abdelnoor M, Svennevig JL. Fifteen-year experience with the Medtronic-Hall valve prosthesis. A follow-up study of 1104 consecutive patients. Circulation 1996; 94: 105-8.

13. Clark RE, Edmunds LH Jr, Cohn LH, et al. Guidelines for reporting morbidity and mortality after cardiac valvular operations. Eur J Cardiothorac Surg 1988; 2: 293-5.

14. Beghi C, De Cicco G, Nicolini F, et al. Cardiac valve reoperations: analysis of operative risk factors in 154 patients. J Heart Valve Dis 2002; 11: 258-62.

15. Husebye DG, Pluth JR, Piehler JM, et al. Reoperation on prosthetic heart valves. An analysis of risk factors in 552patients. J Thorac Cardiovasc Surg 1983; 86: 543-52.

16. Ericsson A, Lindblom D, Semb G, et al. Strut fracture with Björk-Shiley 70 degrees convexo-concave valve. An international multi-institutional follow-up study. Eur J Cardiothorac Surg 1992; 6: 339-46.

17. Bortolotti $U$. The problem of reoperation in bioprosthetic valve recipients. J Heart Valve Dis 1992; 1: 29-31.

18. Kuniyoshi Y, Koja K, Miyagi K, et al. Pannus formation in aortic valve prostheses in the late postoperative period. J Artif Organs 2003; 6: 179-82.

19. Hammermeister K, Sethi GK, Henderson WG, et al. Outcomes 15 years after valve replacement with a mechanical versus a bioprosthetic valve: final report of the Veterans Affairs randomized trial. J Am Coll Cardiol 2000; 36: 1152-8.

20. Van Doorn CA, Stoodley KD, Saunders NR, et al. Mitral valve replacement with the Carpentier-Edwards standard bioprosthesis: performance into the second decade. Eur J Cardiothorac Surg 1995; 9: 253-8.

21. Antunes MJ, Magalhaes MP. Isolated replacement of a prosthesis or a bioprosthesis in the mitral valve position. Am J Cardiol 1987; 59: 346-9.

22. Jamieson WR, Janusz MT, Burr LH, et al. CarpentierEdwards supraannular porcine bioprosthesis: secondgeneration prosthesis in aortic valve replacement. Ann Thorac Surg 2001; 71: 224-7.

23. Girod G, Jaussi A, Rosset C, et al. Cavitation versus degassing: in vitro study of the microbubble phenomenon observed during echocardiography in patients with mechanical prosthetic cardiac valves. Echocardiography 2002; 19: 531-6.

24. Biglioli P, Di Matteo S, Parolari A, et al. Reoperative cardiac valve surgery: a multivariable analysis of risk factors. Cardiovasc Surg 1994; 2: 216-22.

25. Mastroroberto P, Chello M, Bevacqua E, et al. Duromedics original prosthesis: what do we really know about diagnosis and mechanism of leaflet escape? Can J Cardiol 2000; 16: 1269-72.

26. Prasad NK, Alam M, Rosman HS, et al. Serial Doppler gradients are predictive of future bioprosthetic valve degeneration. Echocardiography 1998; 15: 337-44.

27. Antunes MJ. Isolated replacement of a prosthesis or a bioprosthesis in the aortic valve position. Am J Cardiol 1987; 59: 350-2.

28. Turina J, Turina $M$. Left ventricular function and valvular reoperations. J Heart Valve Dis 1995; 4: 223-8.

29. Edwards MB, Ratnatunga CP, Dore CJ, Taylor KM. Thirtyday mortality and long-term survival following surgery for prosthetic endocarditis: a study from the UK heart valve registry. Eur J Cardiothorac Surg 1998; 14: 156-64.

30. de Almeida Brandao CM, Pomerantzeff PM, Souza LR, et al. Multivariate analysis of risk factors for hospital mortality in valvular reoperations for prosthetic valve dysfunction. Eur J Cardiothorac Surg 2002; 22: 922-6.

31. Acar J, Michel PL, Varenne O, et al. Surgical treatment of infective endocarditis. Eur Heart J 1995; 16: 94-8.

32. Akin I, Kische S, Rehders TC, et al. Indication for percutaneous aortic valve implantation. Arch Med Sci 2010; 6: 296-302.

33. Benussi S, Galanti A, Alfieri O. Restoring sinus rhythm in patients at a high risk for postoperative atrial fibrillation. Arch Med Sci 2008; 4: 108-15.

34. Özbek C, Yetkin U, Bademci M, Karahan N, Gürbüz A. Ring annuloplasty and successful mitral valve repair in a staphylococcal endocarditis case with bilobular saccular mycotic aneurysm at cerebral artery and frontal region infarction. Secondary to septic emboli. Arch Med Sci 2008; 4: 94-9. 